\title{
0.60 eV Energy Resolution in EELS Using a Depleted Thermionic LaB -Cathode $^{-}$
}

\author{
C. Mitterbauer***, W. Grogger*, G. Kothleitner*, R. Spehr***, P. Tiemeijer**** and F. Hofer* \\ *Research Institute for Electron Microscopy, Graz University of Technology, Steyrergasse 17, \\ A-8010 Graz, Austria \\ **now at: Department of Chemical Engineering and Materials Science, University of California- \\ Davis, Davis, California 95616, USA \\ ***Institut für Angewandte Physik, Technische Hochschule Darmstadt, Hochschulstrasse 6, \\ D-64289, Darmstadt, Germany \\ ****FEI Electron Optics, PO Box 80066, 5600 KA Eindhoven, The Netherlands
}

The overall energy resolution in Electron Energy Loss Spectroscopy (EELS) recorded in a TEM is a function of the energy spread of the initial electron beam before entering the specimen, the stability of the microscope high tension, the stability of the energy dispersive element of the spectrometer and its electronics, the local environment (mechanical vibrations, temperature and pressure fluctuations as well as parasitic magnetic stray fields), the magnitude of spectral aberrations in the spectrum plane, and the size of the object in the projector cross-over entering the spectrometer [1-4]. Using a thermionic $\mathrm{LaB}_{6}$-source in a Philips CM20 (Scanning) Transmission Electron Microscope ((S)TEM), equipped with a Gatan Imaging Filter (GIF 200) [1] and operated at $200 \mathrm{kV}$, it was ultimately possible to achieve an energy resolution of $0.60 \mathrm{eV}$ measured at the full width halfmaximum (FWHM) of the zero loss peak (ZLP). This ultimate resolution was only obtained with filaments after about 2000 hours of operation.

All spectra were collected in the TEM imaging mode [5] using a small aperture size ( $\beta=7.5 \mathrm{mrad})$ with the $2 \mathrm{~mm}$ spectrometer entrance aperture (SEA) and a high spectrometer dispersion $(0.05$ $\mathrm{eV} /$ pixel). As a compromise between reasonably good counting statistics and overall time of the experiment for core-loss spectra, acquisition times of $400 \mathrm{~s}$ have been chosen. In order to get an estimate for the ZLP resolution at $400 \mathrm{~s}$ the very intense ZLP was acquired in a cumulative way, ensuring not to saturate the 14 bit CCD camera. The FWHM increased from 0.60 to $0.75 \mathrm{eV}$ at 4000 times $0.1 \mathrm{~s}$ (cf. fig.1a). No drift correction was used during the acquisition of the 4000 spectra. With this setup we then verified the energy resolution by measuring the $\mathrm{C} \mathrm{K}$-edge of graphite. It is well known that an energy resolution below $0.8 \mathrm{eV}$ is required to separate the $\sigma^{*}$ double feature at about $292 \mathrm{eV}$ [6]. The $\mathrm{C} \mathrm{K}$ spectrum obtained in our experiment (fig. 1c) features the separation of the $\sigma^{*}$ peak (arrows), which suggested an energy resolution of less than $0.8 \mathrm{eV}$, as indirectly confirmed by the direct ZLP width measurement.

After replacement of the $0.60 \mathrm{eV}$-resolution $\mathrm{LaB}_{6}$-cathode, comparative measurements with a new cathode have been carried out, resulted in a FWHM of $1.10 \mathrm{eV}$ directly after the exchange, $0.85 \mathrm{eV}$ after about 100 hours of cathode lifetime and $0.70 \mathrm{eV}$ after 1900 hours lifetime (fig. 1b) under the same experimental conditions as used before and the same acquisition time of $0.1 \mathrm{~s}$ (cf. fig. 1b) [7]. With a needle-shaped $\mathrm{LaB}_{6}$-cathode ending in a sharp tip on the top side (cf. fig. 1d) it was possible to achieve $0.60 \mathrm{eV}$ energy resolution in EELS at the ZLP with an acquisition time of $0.1 \mathrm{sec}$. The comparison between the estimated Boersch effect from the experimental energy resolution values and calculations of the energy distribution resulting from Boersch effect [8] shows good agreement. So the improved energy resolution can be related to a reduced Boersch effect in beam crossovers due to the increased convergence angle of a depleted $\mathrm{LaB}_{6}$ cathode, reduced emission current and reduced brightness [7]. 


\section{References}

[1] O.L. Krivanek et al., Proc. MAS (1995) 305.

[2] A.J. Gubbens et al., Micron 29 (1998) 81.

[3] H.A. Brink et al., Ultramicroscopy 96 (2003) 367.

[4] G. Kothleitner and F. Hofer, Micron 34 (2003) 211.

[5] R.F. Egerton, EELS in the Electron Microscope, $2^{\text {nd }}$ ed., Plenum Press, New York, 1996.

[6] M.H.F. Overwijk and D. Reefman, Micron 31 (2000) 325.

[7] C. Mitterbauer et al., Micron, to be published.

[8] R. Spehr, Microelectronic Engineering 3 (1985) 61.

[9] This work was supported by the Austrian Science Foundation (FWF). The authors gratefully acknowledge H. Schrö ttner and M. Schmied (both FELMI) for SEM-investigations.
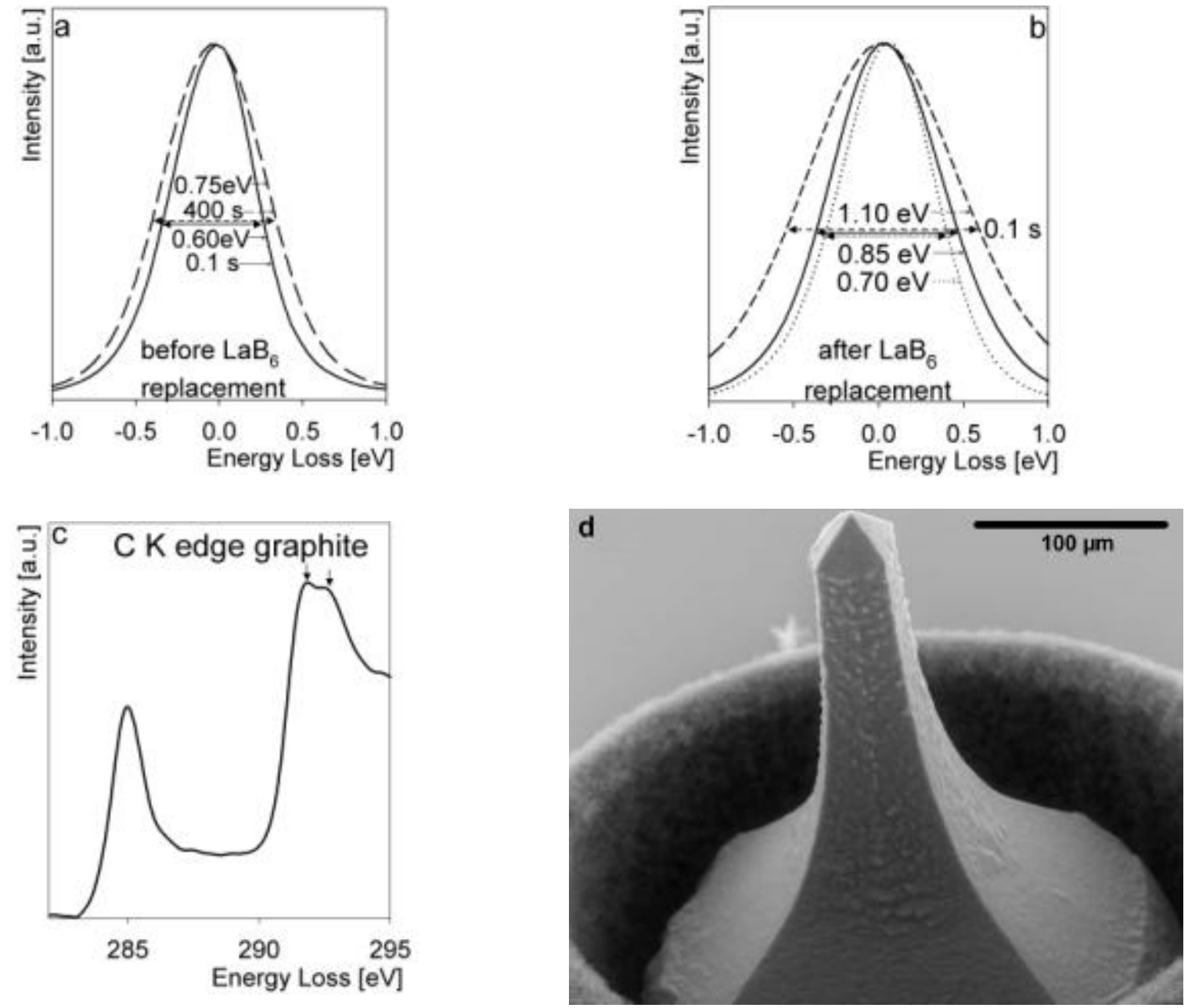

FIG. 1: EELS-measurements demonstrating the energy resolution of the Philips CM20 (+GIF200);

(a) zero-loss spectra before cathode replacement: broken line: $2 \mathrm{~mm}$ SEA and 4000 times $0.1 \mathrm{~s}$ exposure time; solid line: $2 \mathrm{~mm}$ SEA and 0.1s exposure time; (b) zero-loss spectra after different lifetimes of the new cathode; broken line: after $1 \mathrm{~h}$; solid line: after $100 \mathrm{~h}$; dotted line: after $1900 \mathrm{~h}$ (c) the $\mathrm{C} \mathrm{K}$ spectrum recorded with the CM20 and (d) depleted $\mathrm{LaB}_{6}$-cathode after $1900 \mathrm{~h}$ lifetime. 